\title{
Influence of Skull Inhomogeneities on EEG Source Localization
}

\author{
Victoria Montes ${ }^{1}$, Hans Hallez ${ }^{1}$ \\ ${ }^{1}$ Medical Image and Signal Processing, \\ Faculty of Engineering, Ghent University-IBBT \\ Ghent, Belgium \\ victoria.montesrestrepo@ugent.be
}

\author{
Steven Staelens ${ }^{1,2}$ \\ ${ }^{2}$ Molecular Imaging Center Antwerp, \\ Faculty of Medicine, Antwerp University \\ Antwerp, Belgium
}

\begin{abstract}
We investigated the influence of using simplified models of the skull on electroencephalogram (EEG) source localization. An accurately segmented skull from computed tomography (CT) images, including spongy and compact bones as well as some air-filled cavities, was used as a reference model. The simplified models approximated the skull as a homogeneous compartment with: (1) isotropic, and (2) anisotropic conductivity. The results showed that these approximations could lead to errors of more than $2 \mathrm{~cm}$ in dipole estimation. We recommend the use of anisotropy but considering a different ratio for each region of the skull, according to the amount of spongy bone.
\end{abstract}

Keywords-skull conductivity; forward problem; EEG; source localization; anisotropy

\section{INTRODUCTION}

Electroencephalogram (EEG) source analysis relies on an accurate model representing the human head for the computation of the forward solution. In this model, the geometries and conductivities of the different tissues inside the head need to be incorporated. Particularly, the skull presents a low conductivity compared to the other tissues, which has an effect on the surface measurements; therefore, it plays an important role in EEG source localization.

The skull has often been modeled as a homogeneous isotropic compartment. However, the actual structure of the skull has different conductivities and thicknesses and so it is inhomogeneous. Not only compact and spongiform bones are part of its structure but also air-filled cavities such as the paranasal sinuses and mastoid cells. The advances in imaging modalities based on computed tomography (CT) and magnetic resonance (MR) allow segmenting these tissue types as well as constructing a more accurate model of the skull.

The concept of anisotropy was introduced as a way to model the inhomogeneities of the skull. Rush and Driscoll reported the first measurements for the radial and tangential conductivities of the skull in 1968 [1]. Since then, in many applications, the anisotropy ratio of the skull has been assumed to be $10[2,3]$. However, recent studies have suggested that the skull anisotropy must be lower than that [4-6].

The importance of modeling the skull as a layered isotropic structure, accounting for compact and spongy bones, has been pointed out by different authors [6-8]. Nevertheless, their studies have based the skull modeling only on MR images, in

Research is funded by a $\mathrm{PhD}$ grant of the Fund for Scientific Research Flanders (FWO, Belgium). which the bone and its different tissue types are not easily identified.

The objective of this study is to analyze the influence of skull inhomogeneities on EEG source localization. An accurately segmented skull from CT images, including spongy and compact bones as well as some air-filled cavities, is incorporated in the analysis as a reference model. Isotropic and anisotropic conductivity modeling are used as simplified models of the actual layered structure of the skull. The results remark the importance of accurate modeling of the skull on EEG source localization.

\section{METHODS}

\section{A. Head model construction}

Different realistic head models were generated from MR and CT images. The MR images were acquired using a 3T scanner (Siemens Trio, Erlangen, Germany) and consisted of a $256 \times 256 \times 176$ matrix with voxels of $0.9 \mathrm{~mm} \times 0.9 \mathrm{~mm} \times 0.9$ $\mathrm{mm}$. These images were used to segment the scalp and brain tissues. The scalp was segmented through thresholding followed by a closing with hole filling operation. The segmentation of cerebrospinal fluid (CSF), white matter (WM) and gray matter (GM) was done with the SPM8 software [9].

The CT images were acquired with a scanner (Toshiba Aquilion, Tokyo, Japan) and used to accurately segment the skull through a CT/MR gray-value based thresholding approach. Morphological operations such as dilation and erosion were also performed in order to obtain a smooth skull compartment. To distinguish between compact and spongiform bones, a thresholding method based on Gaussian mixture models was employed [10]. The frontal and sphenoidal sinuses were also segmented through thresholding.

The electrode positions were described according to the International 10-20 system, with 6 extra electrodes located at the temporal region, resulting in a total of 27 electrodes. This setup is used for clinical practice at the Department of Neurology of the Ghent University Hospital.

To analyze the influence of the skull on EEG source localization, we constructed three different models:

1) Reference model - Isotropic skull with inhomogeneities: This model consists of separate layers for the compact and spongiform bones of the skull, and includes air- 
filled cavities such as the frontal and sphenoidal sinuses (see Fig. 1). These cavities are assumed to have the conductivity of air. The conductivities for the compact and spongiform bones are chosen according to the measurements of [4] as: $\sigma_{\text {comp }}=$ $0.0064 \mathrm{~S} / \mathrm{m}$ and $\sigma_{\text {spong }}=0.02865 \mathrm{~S} / \mathrm{m}$. Table I shows the conductivity values for the other tissues in the head model.
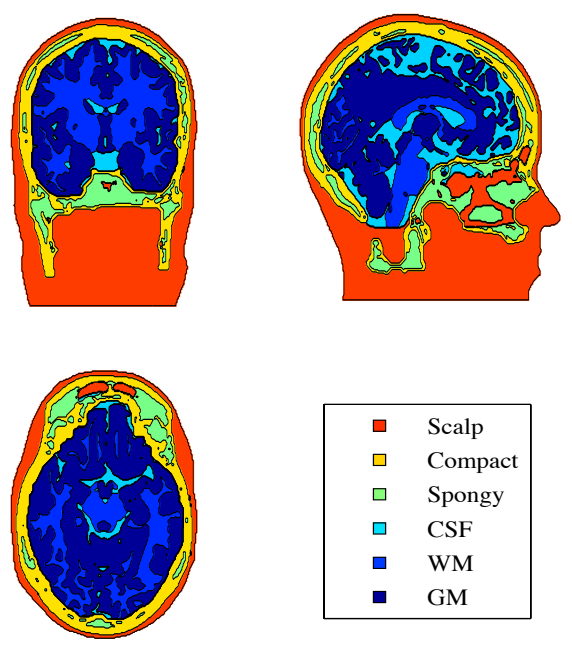

Figure 1. Reference head model in which the segmented tissues can be distinguished.

TABLE I. CONDUCTIVITY VALUES FOR OTHER TISSUES IN THE HEAD MODEL [3]

\begin{tabular}{|l|c|}
\hline \multicolumn{1}{|c|}{ Tissue } & $\begin{array}{c}\text { Conductivity } \\
(\mathbf{S} / \mathbf{m})\end{array}$ \\
\hline Scalp & 0.33 \\
\hline CSF & 1.79 \\
\hline WM & 0.14 \\
\hline GM & 0.33 \\
\hline Air & 0 \\
\hline
\end{tabular}

2) Model 1 - Isotropic skull homogeneous compartment: The skull is modeled as a single compartment, with an isotropic conductivity of $0.020 \mathrm{~S} / \mathrm{m}$ for the whole compartment [11].

3) Model 2 - Anisotropic skull homogeneous compartment: This model incorporates a single anisotropic compartment for the skull surface, and uses its geometry to estimate the radial and tangential conductivities. For each voxel the normal and two orthogonal directions were derived, which in conjunction with the radial and tangential conductivities form the conductivity tensor. The anisotropy ratio of the skull used for this model is based on the works of $[4,8]$ and on our own study on a spherical head model [12]. The radial conductivity is assumed to be $0.0105 \mathrm{~S} / \mathrm{m}$ and the tangential $0.0191 \mathrm{~S} / \mathrm{m}$, i.e., an anisotropy ratio of $1: 1.82$ (radial:tangential) is used.

\section{B. Forward problem}

The forward problem consists in finding the electrode potentials $\mathbf{V}$ at a specified location over the scalp, given a source inside the brain. Sources are modeled as current dipoles, having position $\mathbf{r}$ and orientation $\mathbf{d}$ as their defining parameters. The surface potentials are related to the sources through the Poisson's equation:

$$
\nabla \cdot(\Sigma \nabla V)=\nabla \cdot \mathbf{J}
$$

which is derived from the quasi-static approximation of the Maxwell equations, with Dirichlet and Neumann boundary conditions at each interface inside the head [13]. In (1), $\mathbf{J}$ is the current density imposed by the dipole source and $\Sigma$ is the conductivity tensor or matrix representing the directiondependent conductivity. For isotropic conductivity, $\Sigma$ is a diagonal matrix.

Equation (1) is solved using the Anisotropic Finite Difference with Reciprocity Method (AFDRM) [14], which has been found to be a suitable method for solving the forward problem in a realistic head model with anisotropic compartments, while the grid size is within $1 \mathrm{~mm}^{3}$. With the incorporation of the reciprocity theorem, the potentials are computed for each electrode position rather than for each dipole position. This speeds up the time necessary to do the forward calculations [15].

In this study, the calculation grid of the AFDRM consisted of 5719372 nodes. The time required to compute the forward matrix was approximately 26 hours, for each head model.

\section{Inverse problem}

The inverse problem is defined as the estimation of the dipole parameters $(\mathbf{r}, \mathbf{d})$ that best fit a surface potential. This is done by the minimization of the relative residual energy (RRE) [14]:

$$
R R E=\frac{\left\|\mathbf{V}_{\text {electrodes }}-\mathbf{V}_{\text {model }}(\mathbf{r}, \mathbf{d})\right\|_{2}^{2}}{\left\|\mathbf{V}_{\text {electrodes }}\right\|_{2}^{2}}+C(\mathbf{r})
$$

where $\mathbf{V}_{\text {electrodes }}$ and $\mathbf{V}_{\text {model }}(\mathbf{r}, \mathbf{d})$ are the set of electrode potentials computed in the reference model and the simplified models 1 to 2 , respectively. The term $C(\mathbf{r})$ is a penalization parameter which is zero when the dipole location is inside the GM and large otherwise. The minimization is done by a Nelder-Mead simplex method.

\section{Simulation setup}

Dipole location and orientation errors were investigated when a simplified head model (model $i, \forall i \in 1,2$ ) was assumed instead of a more realistic one (reference model). A similar methodology was followed in $[8,11]$.

Test dipoles were placed on a $3 \mathrm{D}$ grid with distance of 5 $\mathrm{mm}$ between each node. Only the nodes situated in the gray matter and covered by the electrode setup were considered. For each dipole location, three orthogonal orientations were considered according to the Cartesian coordinate system: $\mathrm{X}$-, $\mathrm{Y}-, \mathrm{Z}$-orientation. The total number of test dipoles was 7569 . 
For each test dipole, the following simulation was performed, as seen in Fig. 2:

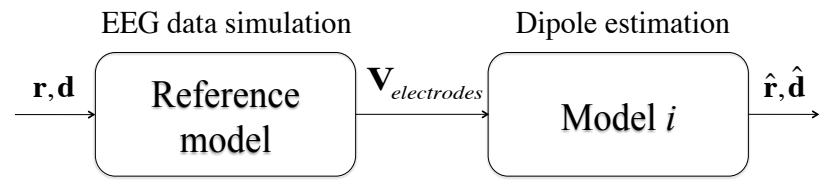

Figure 2. Schematic diagram of the simulation setup.

First, the electrode potentials were calculated by solving the forward problem in the reference model. Thus, the simulated EEG data at the 27 electrodes is obtained.

Subsequently, from the simulated potentials $\mathbf{V}_{\text {electrodes, }}$ the dipoles were estimated by solving the inverse problem using models 1 and 2. In the case of model 1, the skull is modeled as a homogeneous compartment with isotropic conductivity, and in model 2 the conductivity is set to anisotropic. Hence, the error due to the use of a simplified model in the solution of the inverse problem is investigated. The set of dipole parameters, $\hat{\mathbf{r}}$ and $\hat{\mathbf{d}}$ which minimizes the cost function, are the estimated dipole parameters in the simplified head model.

The dipole location error was evaluated through the Euclidean distance between the original dipole location $\mathbf{r}$ and the estimated dipole location $\hat{\mathbf{r}}$ :

$$
D L E=\|\hat{\mathbf{r}}-\mathbf{r}\|
$$

The dipole orientation error, defined as the angle between the vector components of the original dipole $\mathbf{d}$ and the estimated dipole $\hat{\mathbf{d}}$, was calculated through the cosine rule:

$$
D O E=\arccos \left(\frac{\hat{\mathbf{d}}^{T} \mathbf{d}}{\|\mathbf{d}\|\|\hat{\mathbf{d}}\|}\right)
$$

In this way, we can evaluate the dipole localization and orientation errors due to using the following simplified models for the skull: (1) model 1 - isotropic homogeneous compartment, and (2) model 2 - anisotropic homogeneous compartment.

\section{RESULTS}

Fig. 3 shows the results for dipole localization errors on the two simulated head models. Large errors for both models can be seen in the temporal and cerebellar regions. For the isotropic model, the errors are also high in the parieto-occipital region. Although the anisotropic model presents smaller overall errors, the error pattern in temporal and cerebellar regions is similar to that of the isotropic model.

If the right and left hemispheres are compared, asymmetric errors can be seen for both analyzed models. This is due to the higher amount of spongy bone in the left hemisphere on the reference head model (Fig. 1). The histogram of localization errors is shown in Fig. 4. The mean error for model 1-isotropic is $10.5 \mathrm{~mm}$, while for model 2-anisotropic is $6.7 \mathrm{~mm}$.
Fig. 5 displays the dipole orientation errors for both analyzed models. Large orientation errors are shown for dipoles placed in the edges. In the left temporo-occipital region, the error is also high in concordance with the localization errors seen in Fig. 3. According to the histogram, Fig. 6, most dipoles have orientation errors below $20^{\circ}$. The mean orientation error for the isotropic model is $12.7^{\circ}$, and for the anisotropic model is $10.8^{\circ}$.

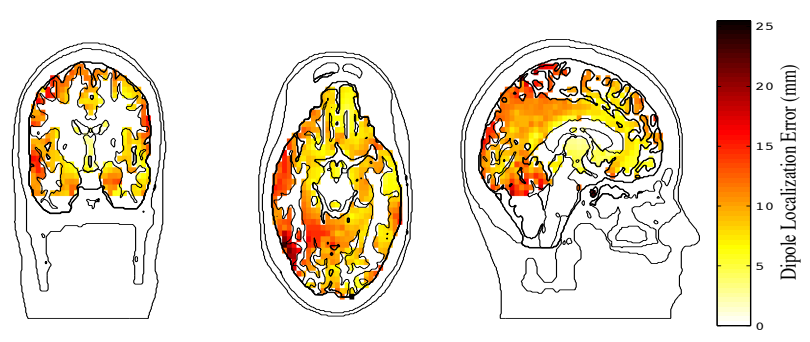

(a) Model 1 - Isotropic homogeneous

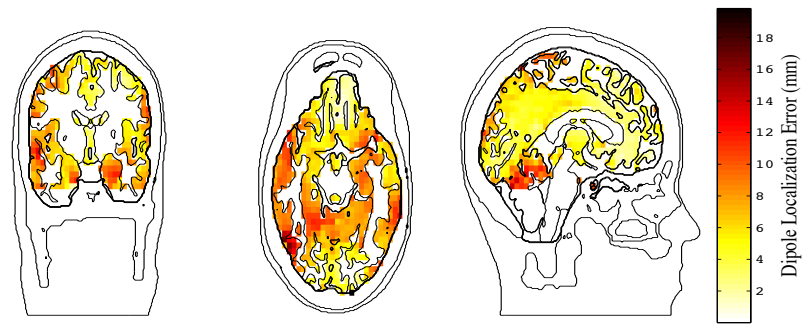

(b) Model 2 - Anisotropic homogeneous

Figure 3. Dipole localization errors.

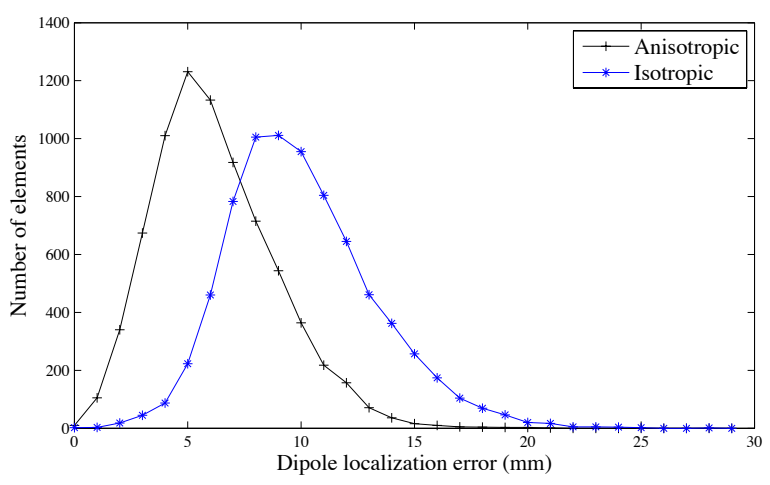

Figure 4. Histogram for the dipole localization errors.

\section{Discussion}

This study analyzed the dipole localization and orientation errors generated when a simplified head model (model $i, \forall i \in$ $1,2)$ was used instead of a more realistic one (reference model), for the inverse problem solution.

We found that modeling the skull as an isotropic homogeneous compartment, with an accurate geometry, can introduce localization errors up to $2.5 \mathrm{~cm}$. Similar results were 
obtained for the isotropic homogeneous model in the study performed by Dannhauer and colleagues [8].

In our model with anisotropic conductivity, an anisotropy ratio of 1.8 was used. According to the smaller errors obtained in the frontal region, we consider that this ratio could be used to model the layered structure of the skull in the regions where the spongy bone has a constant proportion. In other regions such as the temporal, the anisotropy ratio used here did not yield an appropriate model for the layered structure of the skull. The localization errors for this model were up to $1.9 \mathrm{~cm}$. Thus, the anisotropy ratio should be defined for every region according to its structure and spongy bone proportion $[16,17]$.

Despite having defined an accurate geometry for the skull, the present study has some limitations. The reference head model is an approximation by itself due to the lack of precise conductivity values for the skull and its tissue types. In addition, the 27 electrodes setup used here leads to severe spatial subsampling and can generate less accurate localization results [18].

The availability of the different imaging modalities is another limitation. While CT is not routinely done because of the ionizing radiation, in MR the different bone types of the skull are not easily identified.
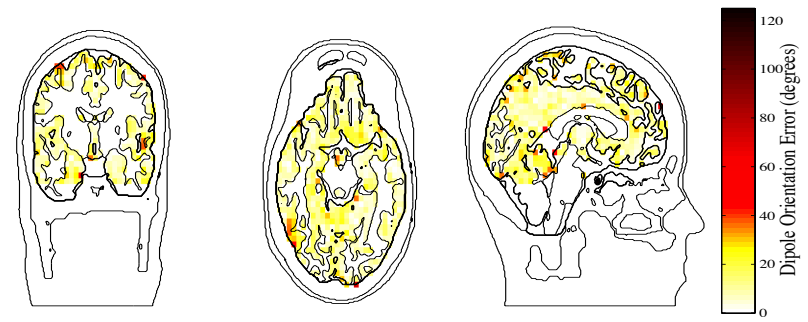

(a) Model 1 - Isotropic homogeneous.
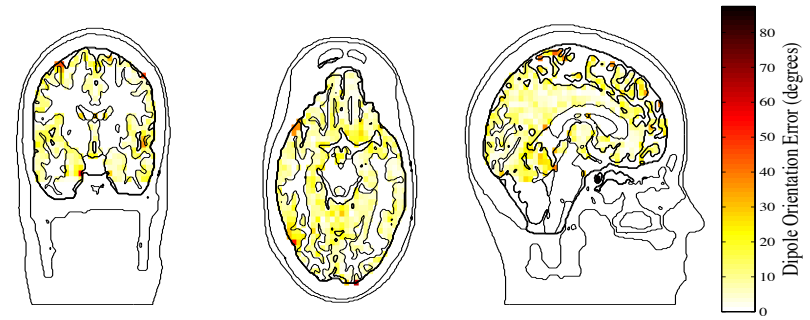

(b) Model 2 - Anisotropic homogeneous.

Figure 5. Dipole orientation errors.

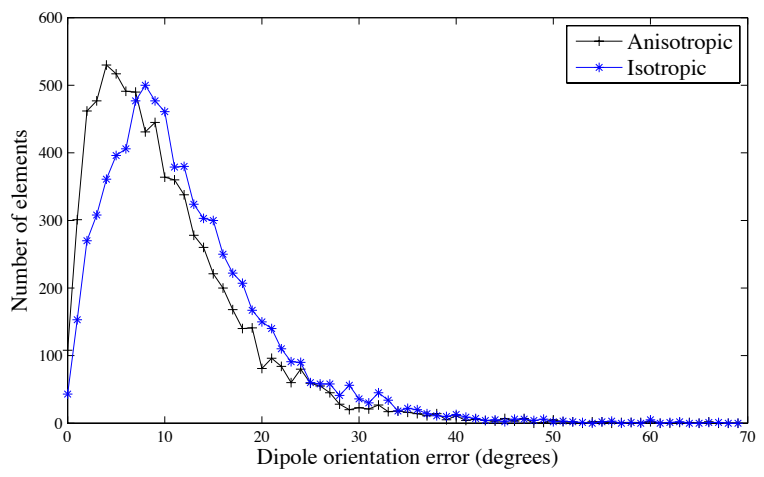

Figure 6. Histogram for the dipole orientation errors.

\section{CONCLUSIONS}

We investigated the dipole location and orientation errors due to assuming a simplified head model instead of a more realistic one in the dipole estimation. The results showed that the approximation of the skull as a homogeneous compartment lead to estimation errors of more than $2 \mathrm{~cm}$. When the conductivity was assumed as isotropic, the errors were up to $2.5 \mathrm{~cm}$. For the case of anisotropic conductivity, the obtained errors were up to $1.9 \mathrm{~cm}$. However, the anisotropy ratio of 1.8 allowed obtaining lower errors in the frontal region of the skull.

Therefore, when the different bone types of the skull cannot be accurately segmented, we recommend the use of anisotropy but assuming different ratios according to the skull region, i.e., taking into account the proportion of spongy bone for that region.

\section{FUTURE WORK}

As a future research, we want to analyze the errors due to incorporating an anisotropic skull with the commonly used ratio of 10. The influence of the air-filled skull cavities on EEG source localization will also be analyzed.

\section{REFERENCES}

[1] S. Rush and D. A. Driscoll, "Current distribution in brain from surface electrodes," Anesthesia and Analgesia, vol. 47, pp. 717-723, 1968.

[2] G. Marin, C. Guerin, S. Baillet, L. Garnero, and G. Meunier, "Influence of skull anisotropy for the forward and inverse problem in EEG: simulation studies using FEM on realistic head models," Human Brain Mapping, vol. 6, pp. 250-269, 1998.

[3] C.H. Wolters et al., "Influence of tissue conductivity anisotropy on EEG/MEG field and return current computation in a realistic head model: A simulation and visualization study using high-resolution finite element modeling," NeuroImage, vol. 30, pp. 813-826, 2006

[4] M. Akhtari et al., "Conductivities of three-layer live human skull," Brain Topography, vol. 14, pp. 151-167, 2002.

[5] M. Fuchs, M. Wagner, and J. Kastner, "Development of volume conductor and source models to localize epileptic foci," Journal of Clinical Neurophysiology, vol. 24, pp. 101-119, 2007.

[6] R. J. Sadleir and A. Argibay, "Modeling skull electrical properties," Annals of Biomedical engineering, vol. 35, pp. 1699-1712, 2007.

[7] R. Pohlmeier et al., "The influence of skull-conductivity misspecification on inverse source localization in realistically shaped 
finite element head models," Brain Topography, vol. 9, pp. 157-162, 1997.

[8] M. Dannhauer, B. Lanfer, C.H. Wolters, and T.R. Knösche, "Modeling of the human skull in EEG source analysis," Human Brain Mapping, doi: $10.1002 / \mathrm{hbm} .21114$.

[9] K.J. Friston, editor. Statistical parametric mapping: The Analysis of Functional Brain Images. Academic Press Inc., U. S., 2006.

[10] Z.-K. Huang and K.-W. Chau, "A new image thresholding method based on Gaussian mixture model", Applied Mathematics and Computation, vol. 205, pp. 899-907, November 2008.

[11] H. Hallez, S. Staelens, and I. Lemahieu, "Dipole estimation errors due to not incorporating anisotropic conductivities in realistic head models for EEG source analysis," Physics in Medicine and Biology, vol. 54, pp. 6079-6093, 2009.

[12] V. Montes, H. Hallez, and S. Staelens, "Determination of anisotropic ratio of the skull for EEG source localization in patients with epilepsy," In Abstracts of the 11th FirW PhD Symposium, Ghent University, pp. 62,2010 .

[13] J. Sarvas, "Basic mathematical and electromagnetic concepts of the biomagnetic inverse problem," Physics in Medicine and Biology, vol. 32, pp. 11-22, 1987.
[14] H. Hallez, B. Vanrumste, P.V. Hese, Y. D’Asseler, I. Lemahieu, and R.V. Walle, "A finite difference method with reciprocity used to incorporate anisotropy in electroencephalogram dipole source localization," Physics in Medicine and Biology, vol. 50, pp. 3787-3806, 2005.

[15] B. Vanrumste, G. Van Hoey, R. Van de Walle, M. D’Have, I. Lemahieu, and P. Boon, "The validation of the finite difference method and reciprocity for solving the inverse problem in EEG dipole source analysis," Brain Topography, vol. 14, pp. 83-92, 2001.

[16] S. K. Law, "Thickness and resistivity variations over the upper surface of the human skull," Brain Topography, vol. 6, pp. 99-109, 1993.

[17] M. R. Bashar, Y. Li, and P. Wen, "Effects of the local skull and spongiosum conductivities on realistic head modeling," In IEEE/ICME International Conference on Complex Medical Engineering (CME2010), Gold Coast, Australia, pp. 23-27, July 2010.

[18] G. Lantz, R. Grave de Peralta, L. Spinelli, M. Seeck, and C. M. Michel, "Epileptic source localization with high density EEG: how many electrodes are needed?," Clinical Neurophysiology, vol. 114, pp. 63-69, 2003. 